Auerbach, M. 5

Bisbe, E. 21

DeLoughery, T.G. 8

Gafter, U. 37

Gafter-Gvili, A. 5, 37, 44, 51

Gasche, C. 30

Gilreath, J.A. 13

Gómez-Ramírez, S. 21

Itzhaki Ben Zadok, O. 51
Jimenez, K.M. 30

Muñoz, M. 21

Rodgers, G.M. 13

Rozen-Zvi, B. 44

Schechter, A. 44

Shander, A. 21

Spahn, D.R. 21

Zusman, O. 51

\title{
Subject Index Vol. 142, No. 1, 2019
}

Ana(e)mia 21, 30, 37, 44, 51

Cancer 13

Chemotherapy-induced anemia 13

Chronic kidney disease 44

Crohn's disease 30

Dialysis 44

Functional iron deficiency 13

Heart failure 51

Hypophosphatemia 8

Inflammatory bowel disease 30

Intravenous iron 13
Iron 8,51

- deficiency 21,30,44

- supplementation 21

Kidney transplant 37

Microbiome 8

Posttransplantation anemia 37

Recombinant erythropoietin 21

Surgery 21

Transfusion 21

Ulcerative colitis 30 